\title{
DESEMPENHO E RENDIMENTO DE CARCAÇA DE FRANGOS ALIMENTADOS COM DIFERENTES FONTES DE SÓDIO
}

\author{
Elizelle Rovatti Rosa, ${ }^{1}$ Débora Cristina Nichelle Lopes, ${ }^{2}$ Aline Arassiana Piccini Roll, ${ }^{3}$ \\ Fabiane Pereira Gentilini, ${ }^{4}$ Victor Fernando Büttow Roll ${ }^{5}$ E Jerri Teixeira Zanusso ${ }^{6}$ \\ 1. Ministério da Agricultura, Pecuária e Abastecimento, Serviço de Inspeção Federal. E-mail: elizelle@gmail.com \\ 2. Mestranda, médica veterinária, PPGZ/DZ/FAEM, UFPel \\ 3. Graduanda, Medicina Veterinária, UFPEL \\ 4. DSc., médica veterinária, GEASPEL/DZ/FAEM, UFPel \\ 5. Engenheiro agrônomo, Dsc. GEASPEL/DZ/FAEM, UFPel \\ 6. PhD., engenheiro agrônomo, professor adjunto, GEASPEL/DZ/FAEM, UFPel
}

RESUMO

Para avaliar os efeitos de diferentes fontes de sódio sobre desempenho e rendimento de carcaça, utilizaram-se 414 fêmeas de corte Ross, de um a 42 dias de idade. O delineamento experimental foi inteiramente ao acaso, com seis repetições e três tratamentos, que consistiam de: T1 - cloreto de sódio; T2 - bicarbonato de sódio com cloreto de amônia; e T3 - formiato de sódio com cloreto de amônia. As variáveis analisadas foram: consumo de ração (CR), ganho de peso (GP), conversão alimentar (CA), viabilidade (VB) e índice de eficiência produtiva (IEP), peso corporal pré-abate $(\mathrm{PCPa})$, rendimento de carcaça $(\mathrm{RC})$, peso da coxa $(\mathrm{PCx})$ e peso de sobrecoxa (PSCx). As aves do T1 e T2 consumiram mais ração $(\mathrm{p}<0,05)$, nos períodos de 1 a 21,1 a 28 e 1 a 35 dias de idade, quando comparadas às do T3. As demais variáveis de desempenho, bem como as de rendimento de carcaça não apresentaram diferença significativa entre os tratamentos. Conclui-se que é possível substituir a fonte clássica de sódio por bicarbonato de sódio e formiato de sódio, fornecidos em conjunto com cloreto de amônia sem prejudicar o desempenho e a qualidade de carcaça de frangos de corte.

PALAVRAS-CHAVES: Consumo, $\mathrm{HCOONa}^{\mathrm{NaCl}}{ }^{-}, \mathrm{NaHCO}_{3}^{-}, \mathrm{NH}_{4} \mathrm{Cl}^{-}$.

ABSTRACT

PERFORMANCE AND CARCASS YIELD OF CHICKENS FED ON THREE DIFFERENT SOURCES OF SODIUM

This study aimed to evaluate the effect of three different sources of sodium for broilers' performance and carcass yield. In total 414 female Ross broilers were fed on dietary treatments from one up to 42 days of age. A completely randomized experimental design was used. Broilers were distributed into a total of 6 replicates per treatment. Treatments consisted of T1- sodium chloride, T2-sodium bicarbonate with Ammonium chloride and T3-sodium formate with Ammonium chloride. Average body weight (ABW), feed consumption (FC), body weight gain (BWG), feed conversion (FE), viability (V) and European efficiency factor
(EEF), preslaughtering body weight (PSBW), carcass yield (CY), thigh yield (TY) and drumstick yield (DY) were evaluated. Broilers fed on $\mathrm{T} 1$ and $\mathrm{T} 2$ showed higher feed conversion than those fed on T3 from 1 to 21,1 to 28 and 1 to 35 days of age. Dietary treatments did not effect the remaining variables up to 35 days of age on the carcass yield. In conclusion, it is possible to substitute the traditional sodium for sodium bicarbonate and sodium formate, both supplied with Ammonium chloride, without affecting the performance or carcass yield.

KEY WORDS: Consumption, $\mathrm{HCOONa}, \mathrm{NaCl}^{-}, \mathrm{NaHCO}_{3}^{-} ; \mathrm{NH}_{4} \mathrm{Cl}^{-}$. 


\section{INTRODUÇÃO}

A dieta de frangos de corte é constituída de ingredientes vegetais, que são deficientes em alguns minerais, destacando-se principalmente o $\mathrm{Na}^{+}$, razão pela qual é necessária a suplementação com produtos que forneçam esse mineral. O cloreto de sódio é o suplemento mineral comumente utilizado, por estar facilmente disponível, por seu baixo custo e pela eficiência em suprir as necessidades de $\mathrm{Na}^{+} \mathrm{e}$ $\mathrm{Cl}^{-}$das aves (BUTOLO et al., 1995). Porém, existem desvantagens quanto ao uso desse produto, principalmente no que envolve as fábricas de rações, tais como grande volume de estocagem, as perdas por corrosão de materiais em virtude de sua característica higroscópica, e perdas, por sua característica oxidativa em relação às vitaminas lipossolúveis contidas nas rações.

Dadas essas desvantagens, tem sido estudada a utilização de bicarbonato de sódio, por amenizar alterações no equilíbrio eletrolítico, evitando prejuízo no desempenho das aves e o aumento da taxa de mortalidade em condições de estresse calórico (MACARI et al., 2002).

Alternativamente ao cloreto de sódio, pode ser usado o formiato de sódio, que, além de suplementar $\mathrm{Na}^{+}$, possui ação antimicrobiana sobre a microflora intestinal, por ser derivado do ácido fórmico (FRANÇA, 2008)

O objetivo deste experimento foi analisar duas fontes alternativas ao cloreto de sódio e avaliar seus efeitos sobre o desempenho e características de carcaça de frangos de corte abatidos aos 42 dias de idade.

\section{MATERIAL E MÉTODOS}

O experimento foi realizado no Aviário Experimental do Departamento de Zootecnia, da Faculdade de Agronomia Eliseu Maciel/UFPEL, no período de 29 de julho a 9 de setembro de 2005 , com temperatura máxima de $20,4^{\circ} \mathrm{C}$ e mínima de $10,4^{\circ} \mathrm{C}$.

Alojaram-se 414 fêmeas de corte, da linhagem Ross, de um dia de idade, em dezoito boxes, totalizando 23 aves/boxe, com peso inicial entre 42 e 50 g. O delineamento experimental foi inteiramente casualizado, com três tratamentos e seis repetições. Representou-se a unidade experimental pelo boxe contendo 23 aves.
Os tratamentos consistiam de diferentes fontes de $\mathrm{Na}^{+}$, sendo $\mathrm{T} 1$ - cloreto de sódio $\left(\mathrm{NaCl}^{-}\right)$; $\mathrm{T} 2$ - bicarbonato de sódio $\left(\mathrm{NaHCO}_{3}^{-}\right)$com cloreto de amônia $\left(\mathrm{NH}_{4} \mathrm{Cl}^{-}\right)$e $\mathrm{T} 3$ - formiato de sódio ( $\mathrm{HCOONa}$ ) com $\mathrm{NH}_{4} \mathrm{Cl}^{-}$. Adicionou-se o $\mathrm{NH}_{4} \mathrm{Cl}^{-}$para ajuste do balanço eletrolítico. As dietas experimentais foram compostas, principalmente, pelo milho e farelo de soja, na forma farelada e oferecidas ad libitum para os períodos de um a 21 dias e 22 a 42 dias de idade dos frangos (Tabela 1).

Utilizou-se o programa de luz recomendado pelo manual da linhagem (AGROCERES ROSS, 2004). As aves e as sobras de ração foram pesadas semanalmente, ocasião em que se avaliaram o consumo de ração (CR), o ganho de peso (GP), a conversão alimentar (CA), a viabilidade (VB) e o índice de eficiência produtivo (IEP).

No $42^{\circ}$ dia de vida, duas aves por boxe foram selecionadas de acordo com a média de peso corporal em cada box $( \pm 5 \%)$, sendo pesadas, anilhadas e levadas para o abatedouro do Conjunto Agrotécnico Visconde da Graça/UFPEL. As características de carcaça avaliadas foram peso corporal pré-abate ( $\mathrm{PCPa})$, rendimento de carcaça $(\mathrm{RC})$, peso da coxa (PCx) e peso da sobrecoxa (PSCx). Submeteram-se os dados à análise estatística mediante o programa estatístico Statistics Analysis System (SAS, 1999), com o procedimento ANOVA, sendo as médias comparadas duas a duas pelo teste de Tukey com nível de significância de $5 \%$.

\section{RESULTADOS E DISCUSSÃO}

Como pode ser observado na Tabela 2, o consumo de ração foi menor $(p<0,05)$ no tratamento contendo formiato de sódio em todos os períodos, exceto de 1 a 42 dias de idade. Esses resultados concordam com FRANÇA (2008), que observou redução do consumo de ração, quando foram usados níveis crescentes de diformiato de sódio na dieta. No estudo de FRANÇA (2008), os níveis de sódio eram crescentes e se aproximavam do ideal. Por isso, a redução do consumo foi atribuída à deficiência de $\mathrm{Cl}^{-}$e ao desequilíbrio ácidobásico influenciados pelos íons de $\mathrm{Na}^{+}, \mathrm{K}^{+}$e $\mathrm{Cl}^{-}$na dieta. $\mathrm{O}$ autor observou também que, quando juntamente com o diformiato de sódio foi adicionado o cloreto de amônia, não houve perdas de desempenho. 
No presente estudo, além da adição de formiato de sódio às dietas, foi adicionado ainda cloreto de amônia. Esta deve ser a razão pela qual não foram observadas diferenças significativas no desempenho das aves em nenhuma das avaliações realizadas, exceto no consumo de ração nos períodos anteriores a 42 dias de idade (Tabela 2).

TABELA 1. Composição percentual e níveis nutricionais das dietas experimentais fornecidas de 1 a 21 dias e de 22 a 42 dias de idade dos frangos de corte

\begin{tabular}{|c|c|c|c|c|c|c|}
\hline \multirow[b]{2}{*}{ Ingredientes (\%) } & \multicolumn{3}{|c|}{1 a 21 dias de idade } & \multicolumn{3}{|c|}{22 a 42 dias de idade } \\
\hline & Cloreto de sódio & $\begin{array}{l}\text { Bicarbonato de } \\
\text { sódio }\end{array}$ & $\begin{array}{l}\text { Formiato de } \\
\text { sódio }\end{array}$ & Cloreto de sódio & $\begin{array}{l}\text { Bicarbonato de } \\
\text { sódio }\end{array}$ & $\begin{array}{l}\text { Formiato de } \\
\text { sódio }\end{array}$ \\
\hline Milho & 52,76 & 52,76 & 52,76 & 57,00 & 57,00 & 57,00 \\
\hline Farelo de soja & 36,74 & 36,74 & 36,74 & 31,52 & 31,52 & 31,52 \\
\hline Óleo de soja & 4,35 & 4,35 & 4,35 & 6,44 & 6,44 & 6,44 \\
\hline Fosfato bicálcico & 1,85 & 1,85 & 1,85 & 1,61 & 1,61 & 1,61 \\
\hline Calcário & 0,90 & 0,90 & 0,90 & 0,82 & 0,82 & 0,82 \\
\hline Sal comum & 0,49 & - & - & 0,44 & - & - \\
\hline Bicarbonato de sódio & - & 0,71 & - & - & 0,63 & - \\
\hline Cloreto de amônia & - & 0,45 & 0,34 & - & 0,40 & 0,30 \\
\hline Formiato de sódio & - & - & 1,16 & - & - & 1,08 \\
\hline Caulin & 1,47 & 0,80 & 0,46 & 1,38 & 0,79 & 0,44 \\
\hline DL-Metionina 98,9\% & 0,10 & 0,10 & 0,10 & 0,08 & 0,08 & 0,08 \\
\hline $\mathrm{L}-\mathrm{Lis} 78,8 \%$ & 0,20 & 0,20 & 0,20 & 0,10 & 0,10 & 0,10 \\
\hline L-Treo & 0,14 & 0,14 & 0,14 & 0,01 & 0,01 & 0,01 \\
\hline Suplemento Vit + Min & $1,00^{1}$ & $1,00^{1}$ & $1,00^{1}$ & $0,60^{2}$ & $0,60^{2}$ & $0,60^{2}$ \\
\hline \multicolumn{7}{|c|}{ Níveis nutricionais calculados } \\
\hline EMAn, kcal/kg & 3000 & 3000 & 3000 & 3200 & 3200 & 3200 \\
\hline Proteína bruta, \% & 21,20 & 21,20 & 21,20 & 19,00 & 19,00 & 19,00 \\
\hline Cálcio, \% & 0,90 & 0,90 & 0,90 & 0,80 & 0,80 & 0,80 \\
\hline Fósforo digestível, \% & 0,45 & 0,45 & 0,45 & 0,40 & 0,40 & 0,40 \\
\hline Potássio, \% & 0,82 & 0,82 & 0,82 & 0,73 & 0,73 & 0,73 \\
\hline Cloro, $\%$ & 0,33 & 0,33 & 0,33 & 0,31 & 0,31 & 0,31 \\
\hline Sódio, \% & 0,21 & 0,21 & 0,17 & 0,19 & 0,19 & 0,16 \\
\hline $\mathrm{BE}, \mathrm{meq} / \mathrm{Kg}$ & 195 & 195 & 177 & 180 & 178 & 165 \\
\hline Arginina digestível, \% & 1,36 & 1,36 & 1,36 & 1,20 & 1,20 & 1,20 \\
\hline Lisina digestível, $\%$ & 1,20 & 1,20 & 1,20 & 1,00 & 1,00 & 1,00 \\
\hline Metionina digestível, & 0,62 & 0,62 & 0,62 & 0,48 & 0,48 & 0,48 \\
\hline $\begin{array}{l}\text { Metionina + cistina dig, } \\
\%\end{array}$ & 0,90 & 0,90 & 0,90 & 0,74 & 0,74 & 0,74 \\
\hline Treonina digestível, \% & 0,85 & 0,85 & 0,85 & 0,65 & 0,65 & 0,65 \\
\hline $\begin{array}{l}\text { Triptofano digestível, } \\
\%\end{array}$ & 0,23 & 0,23 & 0,23 & 0,20 & 0,20 & 0,20 \\
\hline Colina, $\mathrm{mg} / \mathrm{kg}$ & 1292 & 1292 & 1292 & 1167 & 1167 & 1167 \\
\hline Fibra bruta, $\%$ & 2,90 & 2,90 & 2,90 & 2,70 & 2,70 & 2,70 \\
\hline Extrato etéreo, \% & 6,64 & 6,64 & 6,64 & 8,70 & 8,70 & 8,70 \\
\hline
\end{tabular}

${ }^{1}$ Suplemento vitamínico e mineral (garantia): vitamina A: 1.670.000U.I., vitamina D3: 335.000U.I., vitamina E: 2.500mg, vitamina K3: $417 \mathrm{mg}$, vitamina B1: $250 \mathrm{mg}$, vitamina B2: $835 \mathrm{mg}$, vitamina B6: $250 \mathrm{mg}$, vitamina B12: $2.000 \mathrm{mcg}$, ácido fólico: $100 \mathrm{mg}$, biotina: $9 \mathrm{mg}$, niacina: $5.635 \mathrm{mg}$, pantotenato de cálcio: $1.670 \mathrm{mg}$, cobre: $1.000 \mathrm{mg}$, cobalto: $17 \mathrm{mg}$, iodo: $170 \mathrm{mg}$, ferro: $8.335 \mathrm{mg}$, manganês: $10.635 \mathrm{mg}$, zinco: $7.500 \mathrm{mg}$, selênio: $35 \mathrm{mg}$. cloreto de colina: $5.835 \mathrm{mg}$, metionina: $250.000 \mathrm{mg}$, coccidiostático: $13.335 \mathrm{mg}$, promotor de crescimento: $13.335 \mathrm{mg}$, antioxidante: $2.000 \mathrm{mg}$.

${ }^{2}$ Suplemento vitamínico e mineral: vitamina A: 1.335.000U.I., vitamina D3: 300.000U.I., vitamina E: 2.000mg, vitamina K3: 335mg, vitamina B1: $167 \mathrm{mg}$, vitamina B2: $670 \mathrm{mg}$. vitamina B6: 170mg, vitamina B12: $1.670 \mathrm{mcg}$, ácido fólico: $67 \mathrm{mg}$, biotina $7 \mathrm{mg}$, niacina: 4.670mg, pantotenato de cálcio: $1.870 \mathrm{mg}$. cobre: $1.000 \mathrm{mg}$, cobalto: $17 \mathrm{mg}$, iodo: $170 \mathrm{mg}$, ferro: $8.335 \mathrm{mg}$, manganês: $10.835 \mathrm{mg}$, zinco: $7.500 \mathrm{mg}$, selênio: $35 \mathrm{mg}$, cloreto de colina: $41.670 \mathrm{mg}$, metionina: $235.000 \mathrm{mg}$, coccidiostático: $10.000 \mathrm{mg}$. promotor de crescimento: $10.000 \mathrm{mg}$, antioxidante: $2.000 \mathrm{mg}$. 
TABELA 2. Consumo de ração (CR), ganho de peso (GP), conversão alimentar (CA) e viabilidade de frangos de corte alimentados com diferentes fontes de sódio durante os períodos de 1 a 21, 1 a 28, 1 a 35 e 1 a 42 dias de idade

\begin{tabular}{|c|c|c|c|c|c|c|}
\hline Período & Variáveis & & & Tratamento & & \\
\hline \multirow{5}{*}{1 a 21 dias } & & Cloreto de sódio & $\begin{array}{l}\text { Bicarbonato de } \\
\text { sódio }\end{array}$ & Formiato de sódio & Prob & $\mathrm{CV}(\%)$ \\
\hline & CR (g) & $1248,00^{\mathrm{a}}$ & $1244,17^{\mathrm{a}}$ & $1126,83^{b}$ & 0,0124 & 5,72 \\
\hline & GP (g) & 749,17 & 781,17 & 733,00 & 0,1648 & 5,57 \\
\hline & $\mathrm{CA}$ & 1,67 & 1,59 & 1,55 & 0,4203 & 10,08 \\
\hline & VB (\%) & 97,83 & 98,50 & 97,50 & 0,7830 & 2,55 \\
\hline \multirow{5}{*}{1 a 28 dias } & $\mathrm{CR}(\mathrm{g})$ & $2111,00^{\mathrm{a}}$ & $2067,67^{\mathrm{ab}}$ & $1951,83^{\mathrm{b}}$ & 0,0391 & 4,90 \\
\hline & GP (g) & 1359,50 & 1345,50 & 1337,50 & 0,7444 & 3,69 \\
\hline & $\mathrm{CA}$ & 1,55 & 1,54 & 1,46 & 0,0678 & 4,56 \\
\hline & IEP & 314,23 & 318,67 & 331,67 & 0,48543 & 7,89 \\
\hline & VB (\%) & 96,83 & 98,33 & 97,50 & 0,7043 & 3,15 \\
\hline \multirow{5}{*}{1 a 35 dias } & CR (g) & $3278,50^{\mathrm{a}}$ & $3187,00^{\mathrm{ab}}$ & $3044,50^{\mathrm{b}}$ & 0,0351 & 4,43 \\
\hline & GP (g) & 1992,67 & 1961,67 & 1966,50 & 0,6474 & 3,09 \\
\hline & $\mathrm{CA}$ & 1,64 & 1,62 & 1,55 & 0,0695 & 4,19 \\
\hline & IEP & 343,67 & 348,33 & 363,83 & 0,3943 & 7,38 \\
\hline & VB (\%) & 96,67 & 97,67 & 97,50 & 0,8764 & 3,70 \\
\hline \multirow{5}{*}{1 a 42 dias } & $\mathrm{CR}(\mathrm{g})$ & 4161,67 & 4038,83 & 3969,33 & 0,1544 & 4,04 \\
\hline & GP (g) & 2333,50 & 2312,17 & 2344,83 & 0,9413 & 7,07 \\
\hline & CA & 1,79 & 1,76 & 1,70 & 0,6124 & 8,60 \\
\hline & IEP & 309,50 & 318,67 & 330,17 & 0,7534 & 14,78 \\
\hline & VB (\%) & 96,67 & 97,50 & 97,50 & 0,9054 & 3,83 \\
\hline
\end{tabular}

abMédias na mesma linha com letras distintas diferem pelo teste Tukey $(\mathrm{P}<0,05)$

PENZ et al. (1993) afirmaram que os ácidos orgânicos podem proporcionar melhora significativa no aproveitamento dos nutrientes da dieta. $\mathrm{O}$ formiato de sódio poderia interferir nos resultados, dada sua capacidade aniônica tamponante com cátions da dieta $\left(\mathrm{Ca}^{++}, \mathrm{Mg}^{++}, \mathrm{Fe}^{++}, \mathrm{Cu}^{++}, \mathrm{Zn}^{++}\right)$, aumentando, assim, a digestibilidade e retenção desses elementos no organismo. Os ácidos orgânicos poderiam reduzir o $\mathrm{pH}$ do trato gastrintestinal (PARTANEN et al., 2001). A redução de $\mathrm{pH}$ no trato gastrintestinal pode aumentar a atividade de certas enzimas e melhorar a digestibilidade de alguns nutrientes da dieta (PENZ et al., 1993).

Neste experimento não foram analisadas as variáveis respostas que poderiam confirmar essa afirmação, tais como $\mathrm{pH}$ da dieta e do trato digestivo das aves. Porém a inexistência de diferenças significativas entre os tratamentos indicam que essas propriedades não foram suficientes para melhorar o desempenho das aves.

Outra propriedade atribuída aos ácidos orgânicos é que eles poderiam inibir o desenvolvimento microbiano, afetando as funções vitais como, por exemplo, síntese de macromoléculas, $\mathrm{pH}$ do citoplasma e transporte de substrato (CHERRINGTON et al., 1991)

FRANÇA (2008) verificou que o uso de diformiato de sódio como fonte exclusiva de $\mathrm{Na}^{+}$aos sete dias de idade reduziu o consumo de água e no período de 1 a 21 dias de idade prejudicou o desempenho frangos de corte.

Segundo MAIORKA et al. (1998), o consumo de alimento aumenta com o consumo de água, que, por sua vez, aumenta com a quantidade de sódio na dieta. Nesse sentido, BORGES et al. (1999) explicaram que há aumento no consumo de água porque as aves tentam satisfazer a sensação de sede criada pelo aumento na ingestão de sódio.

Neste experimento não foi avaliado o consumo de água, razão pela qual não se pode corroborar tal afirmação. No entanto, não se descarta a hipótese de que a redução no consumo nas dietas contendo formiato explica-se que em parte pelo forte odor ácido, que poderia reduzir a sua palatabilidade. CANIBE et al. (2001) relataram que as propriedades físico-químicas 
dos ácidos podem afetar a palatabilidade da dieta para suinos, limitando os níveis de inclusão nela. Da mesma forma, YAJIMA (2002) observou redução do consumo diário de ração em 4,1 gramas em poedeiras que receberam ração com adição de $0,1 \%$ de acidificante.

De acordo com os dados apresentados na Tabela 2, observa-se que a adição do formiato e do bicarbonato de sódio não prejudicou o IEP, apoiando a ideia de que as variáveis de desempenho mais importantes, que são viabilidade, peso vivo, idade de abate e conversão alimentar, não foram afetadas pelas diferentes fontes de sódio. Portanto, estes resultados indicam que podem ser alternativas economicamente viáveis ao uso tradicional do cloreto de sódio.

A viabilidade esteve em média dentro do que é recomendado pelo guia de manejo da linhagem de frangos de corte estudada. BRANTON et al. (1986) observaram redução da mortalidade em aves expostas a estresse calórico quando alimentados com dietas contendo $\mathrm{NaHCO}_{3}^{-}$. O bicarbonato de sódio tem sido usado para aumentar o consumo de água, bem como para aumentar a ingestão de íons específicos, preve- nindo mudanças no equilíbrio ácido-básico e aliviando os efeitos negativos das altas temperaturas sobre o desempenho e a sobrevivência das aves (MACARI et al., 2002). Neste experimento não foram encontradas tais diferenças, provavelmente porque não houve um estresse calórico, pelo fato de o experimento ter sido desenvolvido durante o inverno.

É possível também fornecer o bicarbonato de sódio em conjunto com o $\mathrm{NaCl}$ sem reduzir o desempenho de frangos de corte de 21 a 42 dias de idade (FISHER DA SILVA et al., 2000).

O fornecimento de formiato de sódio $(0,17 \%$ de sódio) não afetou as variáveis de rendimento de carcaça (Tabela 3). Esses resultados são compatíveis com os resultados encontrados por MURAKAMI et al. (2000), que, ao avaliarem as exigências de sódio e cloro para frangos de corte com idades de 42 e 56 dias, fornecendo duas fontes de sódio $\left(\mathrm{NaCl}^{-} \mathrm{e} \mathrm{NaHCO}_{3}{ }^{-}\right)$, observaram que o nível de $0,15 \%$ foi suficiente para suprir as exigências dos animais, mantendo o peso corporal máximo e uma melhor conversão alimentar.

TABELA 3. Médias das variáveis de carcaça peso corporal pré-abate (PCPa, g), rendimento de carcaça (RC, \%), peso de coxa (PCx, g) e peso de sobrecoxa (PSCx, g) para as diferentes fontes de sódio

\begin{tabular}{lcccc}
\hline Tratamento & PCPa & RC & PCx & PSCx \\
\hline $\mathrm{NaCl}^{-}(\mathrm{T} 1)$ & 2433,83 & 81,96 & 239,50 & 257,50 \\
$\mathrm{NaHCO}_{3}^{-}(\mathrm{T} 2)$ & 2400,83 & 81,17 & 233,00 & 251,17 \\
Formiato de $^{+}$(T3) & 2409,50 & 82,01 & 232,67 & 257,67 \\
Prob & 0,8270 & 0,3788 & 0,4335 & 0,7019 \\
$\mathrm{CV}(\%)$ & 3,96 & 1,40 & 4,27 & 5,90 \\
\hline
\end{tabular}

BARROS et al. (2004) encontraram exigências de sódio de $0,197 \%$ para machos e $0,317 \%$ para fêmeas na fase de crescimento. Já para fase final, foram de $0,216 \%$ e $0,245 \%$, respectivamente, sendo as exigências mais altas que as observados neste experimento.

Quanto aos ácidos orgânicos, os resultados observados concordam com os encontrados por IZAT et al. (1990), GARCIA et al. (2000), RIBEIRO et al. (2001), CAMPOS et al. (2004), MAIORKA et al. (2004), CUNHA et al. (2006), que não observaram perdas em nenhuma das variáveis de desempenho, por ocasião da utilização destes na forma isolada ou combinada em dietas de frangos de corte.

\section{CONCLUSÃO}

É possível substituir a fonte clássica de sódio $\left(\mathrm{NaCl}^{-}\right)$por bicarbonato de sódio $\left(\mathrm{NaHCO}_{3}^{-}\right)$e formiato de sódio (HCOONa), fornecidos em conjunto com cloreto de amônia, sem prejudicar o desempenho e a qualidade de carcaça de frangos de corte. 


\section{REFERÊNCIAS}

AGROCERES ROSS. Manual de manejo de frangos AGROSS, 2004. Campinas: Produção e Editoração UmDesign, 2004. 119 p.

BARROS, J. M. S.; GOMES, P. C.; ALBINO, L. F. T.; ROSTAGNO, H. S.; COSTA, L. F. Exigência de sódio para frangos de corte nas fases de crescimento ( 22 a 42 dias) e final (43 a 53 dias). Revista Brasileira Zootecnia, v. 33, n. 6, 2004. Disponível em: $<$ http://www.scielo.br/scielo.php?script=sci_arttext\&pid=S1516$35982004000700011 \& \operatorname{lng}=$ pt\&nrm=iso $>$. Acesso em: $1 .^{\circ} \mathrm{dez}$. 2006.

BORGES, S. A.; ARIKI, J.; SANTIN, E.; FISCHER DA SILVA A. V.; MAIORKA, A. Balanço eletrolítico em dieta pré-inicial de frangos de corte durante o verão. Revista Brasileira de Ciência Avícola, v. 1, p. 175-179, 1999.

BRANTON, S. L., REECE, F. N.; DEATON, J. W. Use of ammonium chloride and sodium bicarbonate in acute heat exposure of broilers. Poultry Science, v. 65, p. 1659-1666, 1986.

BUTOLO, E. A. F.; NOBRE, P. T. C.; LIMA, I. A. Estudo do desempenho de frangos de corte alimentados com diferentes níveis de cloreto de sódio $(\mathrm{NaCl})$. In: CONFERÊNCIA APINCO DE CIÊNCIA E TECNOLOGIA AVÍCOLAS, 95., 1995, Curitiba, Anais... Campinas, SP. 1995, p. 51-52

CAMPOS, M. A. P.; RABELLO, C. B. V.; SAKOMURA, N.K.; LONGO, F. A.; KUANA, S.; GUT, F. Utilização do ácido fumárico em dietas de frangos de corte com baixa energia metabolizável. Acta Scientiarum. Animal Science. v. 26, n. 1, p. 35-39, 2004. Disponível em: <http://www.ppg.uem.br/docs/ctf/ Zootecnia/2004_1/06_349_03_Maida\%20acido\%20fumarico\%20 Resumo.pdf $>$ Acesso em: 23 jan. 2007.

CANIBE, N. STEIEN, S. H.; OVERLAND, M.; JENSEN, B. B. Effect of K-diformate in starter diets on acidity, microbiota, and the amount of organic acids in the digestive tract of piglets and on gastric alterations. Journal Animal Science, v. 79, p. 21232133, 2001.

CHERRINGTON, C. A.; HINTON, M.; CHOPRA, I. Organic acids: chemistry, antibacterial activity and practical applications. Advances Microbiological Physiology, v. 32, p. 87-108, 1991.

CUNHA, F.; OPALINSKI, M.; MAIORKA, A.; DAHLKE, F.; FRANÇA, M. I.; KRABBE, E. L. Avaliação do formiato de sódio em substituição ao cloreto de sódio em dietas de frangos de corte. Revista Brasileira de Ciência Avícola, Suplemento 8, p. $65,2006$.

FISCHER DA SILVA, A. V; FLEMMING, J. S.; BORGES, S. B. Fontes de sódio e relação sódio: cloro para frangos de corte. Revista Brasileira de Ciência Avícola, v. 2, p. 52-58, 2000.
FRANÇA, M. I. Uso de formiato de sódio e potássio em rações para frangos. 2008. 53 f. Dissertação (Mestrado em Ciências Veterinárias) - Setor de Ciências Agrárias, Universidade Federal do Paraná, Curitiba, 2008.

GARCIA, R. G.; ARIKI, J.; MORAES, V. M. B.; KRONKA, S. N.; BORGES, S. A.; MURATA, L. S.; CAMPOS, V.A. Ação isolada ou combinada de ácidos orgânicos e promotor de crescimento em rações de frangos de corte. Revista Brasileira de Ciência Avícola, Campinas, v. 2, n. 2, 2000. Disponível em: <http://www.scielo.br/ scielo.php?script $=$ sci_arttext\&pid $=$ S1516635X2000000200004\& lng=pt\&nrm=iso $>$. Acesso em: 4 dez. 2006.

IZAT, A. L.; ADAMS, M. H.; CABEL, M. C.; COLBERG, M.; REIBER, M. A.; SKINNER, J. T., WALDROUP, P.W. Effects of formic acid or calcium formate in feed on performance an microbiological characteristics of broilers. Poultry Science, v. 69, p. 1876-1882, 1990.

MACARI, M.; FURLAN, R.L.; GONZÁLES, E. Equilíbrio ácido-básico. In: Fisiologia aplicada a frangos de corte. Jaboticabal: FUNEP/ UNESP, 2002. p. 51-74.

MAIORKA, A.; MAGRO, N.; BARTELS, H.A.; PENZ JR., A. M. Efeito do nível de sódio e diferentes relações entre sódio, potássio e cloro, em dietas pré-iniciais no desempenho de frangos de corte. In: REUNIÃO ANUAL DA SOCIEDADE BRASILEIRA DE ZOOTECNIA, 1998, Botucatu, Anais... Botucatu, 1998. p. 478-480.

MAIORKA, A.; SANTIM, A. M. E.; BORGES, S. A.; OPALINSKI, M.; SILVA, A.V.F. Emprego de uma mistura de ácidos fumárico, lático, cítrico e ascórbico em dietas iniciais de frangos de corte. Archives of Veterinary Science, v. 9. n. 1, p. 31-37. 2004.

MURAKAMI, A. E. ; SALEH, E. A.; WATKINS, S.E.; WALDROUP, P. W. Sodium source and level in broiler diets with and without high levels of animal protein. The Journal of Applied Poultry Research, v. 9, p. 53-61, 2000.

PARTANEN, K., JALAVA, T.; VALAJA J.; PERTTILÄ, S.; SILJANDER-RASI, H., LINDEBERG. H. Effect of dietary carbadox or formic acid and fibre levbel on ileal and faecal nutrient digestibility and microbial metabolite concentrations in ileal digesta of the pig. Animal Feed Science and Technology, v. 93, p. $137-155,2001$.

PENZ, Jr. A. M.; SILVA, A. B.; RODRIGUES, O. Ácidos orgânicos na alimentação de aves. In: CONFERÊNCIA APINCO DE CIÊNCIA E TECNOLOGIA AVÍCOLAS, Santos Anais... São Paulo, Brasil: FACTA 1993. p. 111-119.

RIBEIRO, A. M. L.; MAHMOUD, H.; TEETER, R. G.; PENZ, Jr. A. M. Avaliação das propriedades do ácido nicotínico no desempenho e no balanço térmico de frangos de corte durante estresse por calor. Revista Brasileira de Ciência Avícola, v. 3, n. 1, 2001, Disponível em: <http://www.scielo.br/scielo.php?script=sci_ 
arttext\&pid=S1516-635X2001000100004\&lng=pt\&nrm=iso $>$. Acesso em: 23 jan. 2007.

SAS INSTITUTE. Statistics Analysis System. Cary, N.C.: SAS Institute Inc., 1999.
YAIJIMA, H. H. et al. Avaliação de acidificante em aves de postura comercial. Arquivo Instituto Biológico, v. 69 (supl.) p. 135-135, 2002.

Submetido em: 31 jul. 2007. Aceito em: 1. ${ }^{\circ}$ fev. 2010. 